\title{
1 Sensory substitution reveals a manipulation bias
}

2 Authors: AT Zai ${ }^{1,3}$, S Cavé-Lopez ${ }^{1}$, M Rolland ${ }^{2}$, N Giret ${ }^{2}$, RHR Hahnloser ${ }^{1,3,{ }^{*}}$

4 Affiliations:

$5 \quad{ }^{1}$ Institute of Neuroinformatics, University of Zurich and ETH Zurich, 8057 Zurich, Switzerland

$6 \quad$ Institut des Neurosciences Paris Saclay, UMR 9197 CNRS, Université Paris Sud, Université Paris Saclay,

7 Orsay, France.

$8{ }^{3}$ Neuroscience Center Zurich (ZNZ), University of Zurich and ETH Zurich, 8057 Switzerland.

$9{ }^{*}$ Correspondence to: rich@ini.ethz.ch

11 Abstract:

12 Sensory substitution is a promising therapeutic approach for replacing a missing or diseased sensory

13 organ by translating inaccessible information into another sensory modality. What aspects of

14 substitution are important such that subjects accept an artificial sense and that it benefits their

15 voluntary action repertoire? To obtain an evolutionary perspective on affective valence implied in

16 sensory substitution, we introduce an animal model of deaf songbirds. As a substitute of auditory

17 feedback, we provide binary visual feedback. Deaf birds respond appetitively to song-contingent visual

18 stimuli, they skillfully adapt their songs to increase the rate of visual stimuli, showing that auditory

19 feedback is not required for making targeted changes to a vocal repertoire. We find that visually

20 instructed song learning is basal-ganglia dependent. Because hearing birds respond aversively to the

21 same visual stimuli, sensory substitution reveals a bias for actions that elicit feedback to meet animals'

22 manipulation drive, which has implications beyond rehabilitation. 
Sensory substitution is a transformation of stimuli from one sensory modality into another one ${ }^{1}$. Such transformation can be used as a therapeutic approach towards restoring perception from a defective sensory modality ${ }^{2}$. This approach has gained much interest in recent years thanks to both advances in technology and the remarkable cross-modal flexibility of the central nervous system ${ }^{3-5}$. However, one of the main obstacles hindering the wide adoption of substitution devices has been the amount of training necessary to make use of the new sensory input; in fact, blind subjects often give up using a substitution device before reaching a reasonable proficiency level because they feel overwhelmed and frustrated ${ }^{4}$. inherent in sensory substitution are poorly understood, partly because we are lacking a theory that would predict how a subject will respond to substituting input. One key question is whether substitution will increase or decreases the affective valence of a given motor action ${ }^{6,7}$. Ideally, we would like to know beforehand about actions that will suffer from a decrease in valence and therefore will be avoided by subjects. Vice versa, if we could predict the actions that will experience a boost in valence from substitution, we could provide better treatments to support skilled behaviors such as speech in the deaf. sensorially deprived subjects desire highly informative feedback about their actions. For example,

42 substituting input could help subjects to reduce uncertainties inherent in their motor output and allow

43 them to make better action choices. Accordingly, the artificial sensory input should perfectly

44 differentiate among distinct action outcomes. Formally, substitution may elicit the desire to explore ${ }^{8-10}$, 45 which is to seek knowledge about actions' effects. According to this knowledge seeking view, subjects will preferentially choose actions with uncertain outcomes ${ }^{11}$ or high predicted information gain ${ }^{12-14}$. 
47 Another idea is that adaptive responses to substitution may focus on the intrinsic goal of manipulating

48 the environment ${ }^{15}$ rather than to obtain knowledge. For example, subjects may be drawn towards

49 actions for the sole reason that the latter trigger a significant sensory input. Substitution could thus

50 reveal a drive for impact ${ }^{16}$, which is to preferentially choose actions with noticeable effect.

51 To test whether knowledge-seeking or impact-seeking better explains adaptive responses to sensory

52 substitution, in songbirds we partially replace auditory feedback from a complex vocal behavior by visual

53 feedback. We modified a widely applied operant conditioning paradigm involving the pitch of a song

54 syllable. Instead of using short white-noise bursts played through a loudspeaker ${ }^{17,18}$, we substitute

55 auditory by visual feedback by briefly switching off the light in the sound-isolation chamber of the

56 singing bird whenever the pitch of a targeted syllable was below (or above) a threshold, Fig. 1. We set

57 the pitch threshold for light-off every morning to the median pitch value on the previous day. We

58 investigated whether adult male zebra finches deafened using bilateral cochlea removal would show any

59 kind of reaction in response to such pitch substitution by light-off (LO). We evaluated bird's responses to

60 substitution in terms of $d^{\prime}$ values, which are average daily pitch changes normalized by their standard

61 deviations (see Methods). From these values, we inferred the affective valence of substituted feedback:

62 whether it is neutral, aversive, or appetitive. 
a

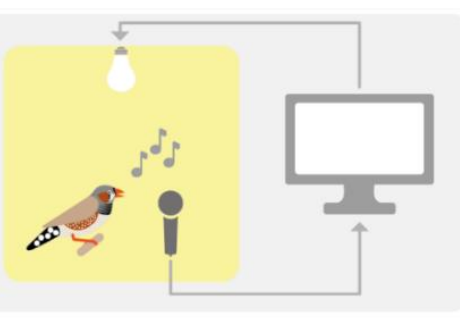

c
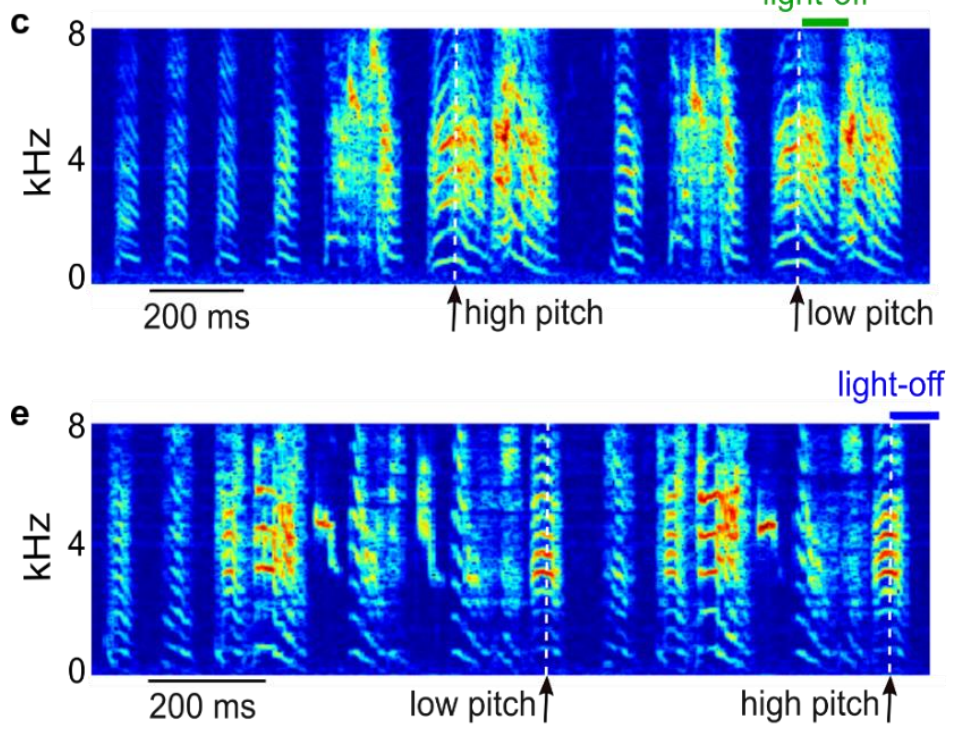

light-off

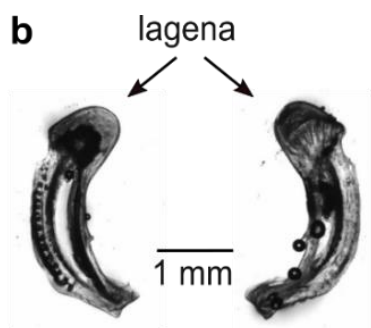

63

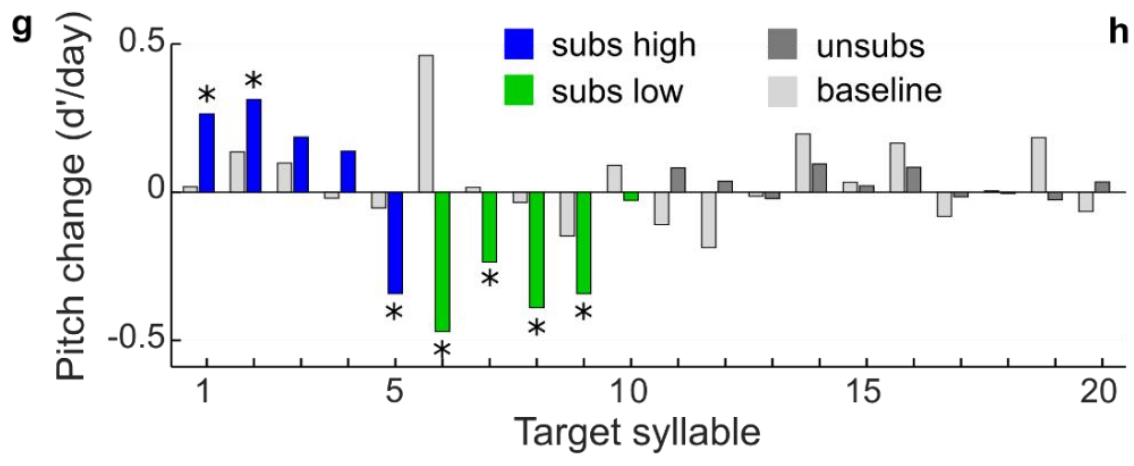

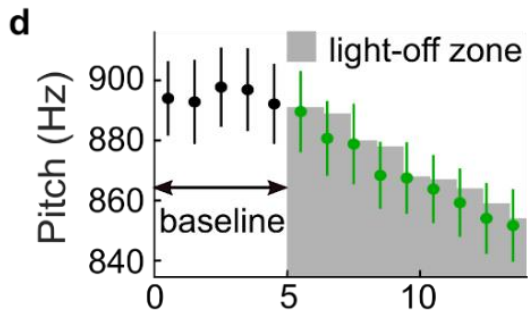

Days since deafening

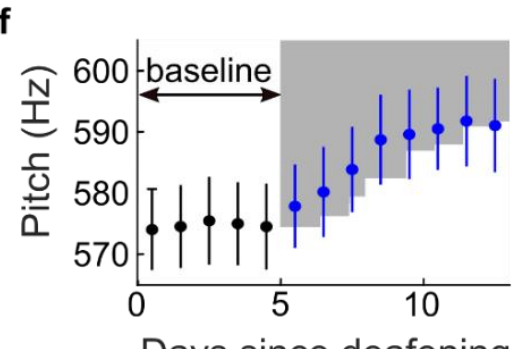

Days since deafening

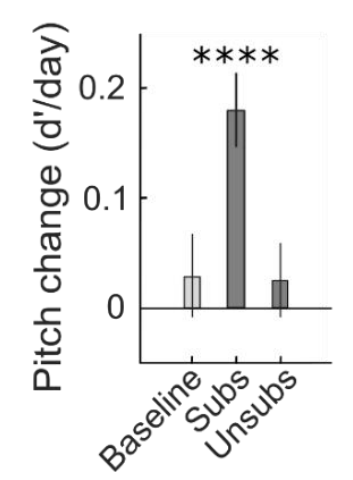

Fig. 1: Light-off stimuli are positive reinforcers of vocal pitch in deaf songbirds. a, Schematic of the

experiment. A singing deaf bird inside a sound isolation chamber (left) experiences light off for a duration in the range of 100-500 ms (right) when the pitch of one of its song syllables (red note) exceeds a given threshold. $\boldsymbol{b}$, Example picture of a pair of surgically removed cochlea. Complete deafness was confirmed by presence of the osseous spiral lamina and by verification of an intact loop including the lagena. $c, e$, Example song spectrograms in birds b2y2 (c) and b2p19 (e) with substituted feedback for low-pitched (c) 
and high-pitched (e) syllable renditions (the time points of pitch measurement are indicated by white

71

dashed lines). $\boldsymbol{d}, \boldsymbol{f}$, Daily mean pitches (dots) and their standard deviations during baseline (black) and during substitution (green: low-pitch subs, $d$; blue: high-pitch subs, f). The light-off pitch zones are indicated by gray shading. The birds adapted the pitch in the direction of increasing light-off rate. $\boldsymbol{g}$, Histograms of average daily pitch changes during substitution in birds with high-pitch substitution (subs high, blue, $n=5$ birds, the first bar corresponds to b2p19 shown in e and f), low-pitch substitution (subs low, green, $n=5$ birds, the $8^{\text {th }}$ bar corresponds to b2y2 shown in $c$ and d), and in control birds without substitution (unsubs, dark grey, $n=7$ birds, target syllables 11 and 14 stemmed from the same bird and so did syllables $13+18$ and $15+19)$. The light grey bars to the left of the colored bars indicate the average daily pitch change for each syllable during the last 5 baseline days. The asterisks indicate subs birds with significant pitch changes compared to controls (two sample, two-sided t-test, $p<0.05$ ). $\boldsymbol{h}$, Shown are the three fixed-effect terms of a mixed linear effect model and their standard errors (see main text for details). The bars indicate the daily change in pitch (d'/day) during baseline (left, $p=0.44)$, during substitution in the direction of increasing light-off rate (subs, $p=2.0^{*} 10^{-7}$ ), and in control birds (unsubs, right, $p=0.46)$.

\section{Substituted feedback appetitively reinforces vocal pitch}

Because deafening by itself may induce a slow pitch drift with a nonzero bias ${ }^{19,20}$, we evaluated pitch responses in comparison to unsubstituted deaf control (unsubs) birds. Pitch changes in $7 / 10$ subs birds significantly deviated from the changes in control animals in matched time periods ( $p<0.05$ in 7 of 10 subs birds, two sample, two-tailed t-test of pitch change per day, see Methods, Fig. 1g).

Interestingly, subs birds tended to be attracted by light-off, because all birds except one changed their pitch in the direction of increasing LO rate, Fig. 1g. If the direction of pitch drift were random in each 
93 bird with probability $1 / 2$ in each direction (binomial model), then 9 of 10 birds would drift in the same

94 direction in less than $1 \%$ of cases, corresponding to a p-value smaller than 0.01 , whence the attraction

95 was a non-random effect.

96 To account for individual variability, we fitted mixed linear effect models to the pitch data. The models

97 contained three fixed terms: one term for the early time period before substitution and one term each

98 for the late time periods in subs and unsubs birds. In addition, there was one random term for each

99 syllable ( $n=17$ birds, 20 target syllables, see Methods). We found that relative to baseline (early period),

100 subs birds exhibited pitch changes of $0.18 \mathrm{~d} /$ day in the direction of increasing LO rate (nonzero fixed

101 effect, $p=2.0 * 10^{-7}, S E=0.03$, tstat=5.33, DF=282, Fig. $1 \mathrm{~h}$ ), whereas unsubs birds did not change pitch

$102\left(0.02 d^{\prime} /\right.$ day, $p=0.46, S E=0.03$, tstat $\left.=0.74, D F=282\right)$.

103 Syllables in deaf birds remained stable over the short period of the experiment; changes were specific to 104 pitch but did not affect other sound features ( $p>0.05$, two-tailed t-test, duration, frequency modulation, 105 amplitude modulation, and entropy, see Methods), see Supplementary Fig. S1. In combination, these 106 results indicate that in deaf birds, substituted feedback is an appetitive reinforcer of song. 
a

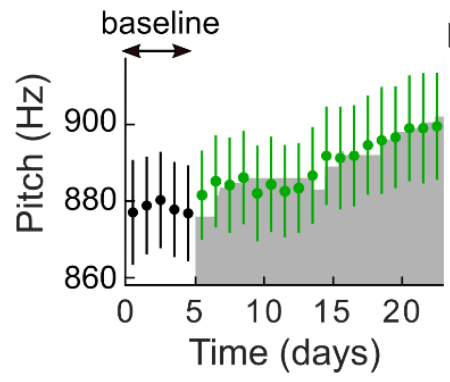

b

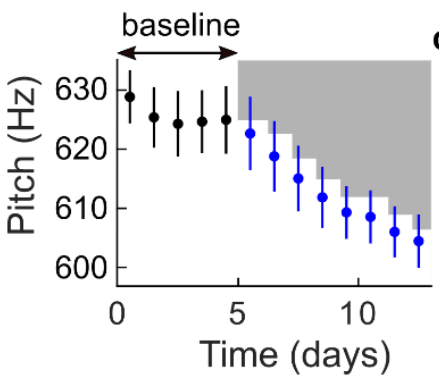

d

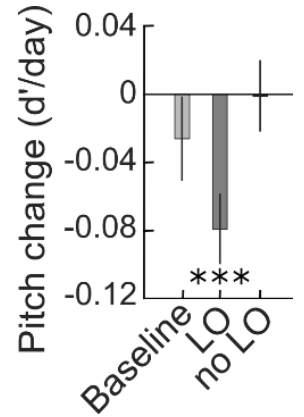

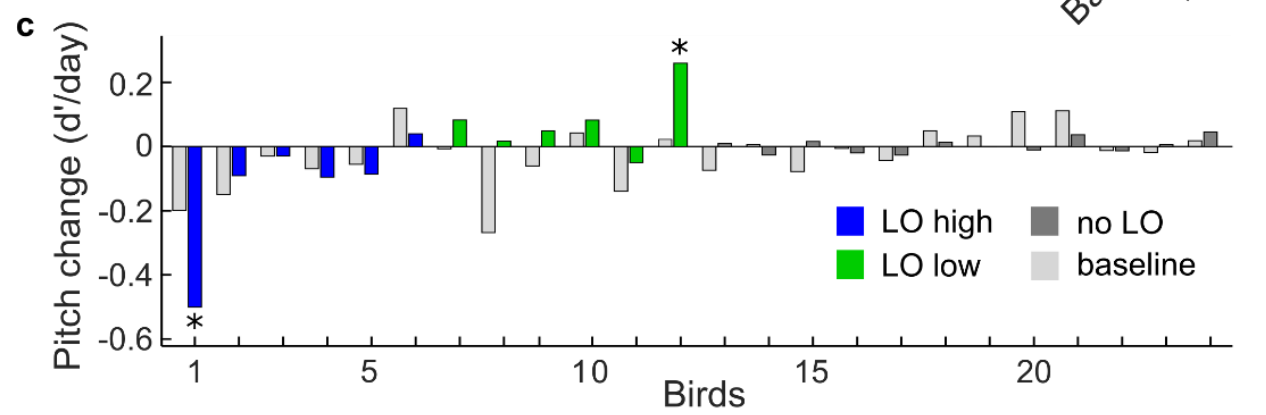

e
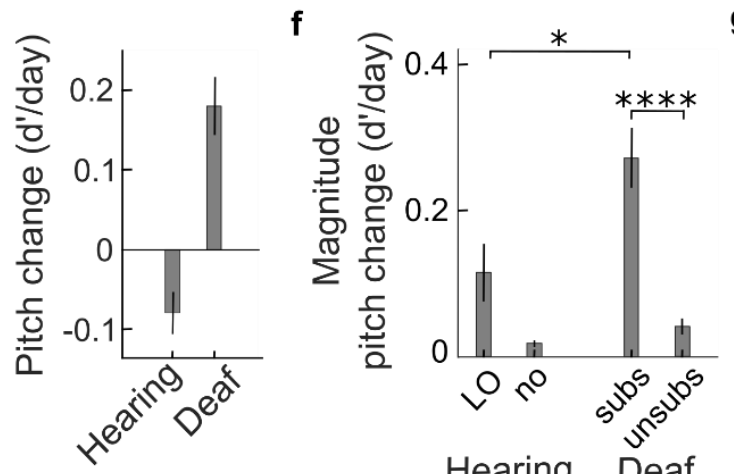

Hearing Deaf

g

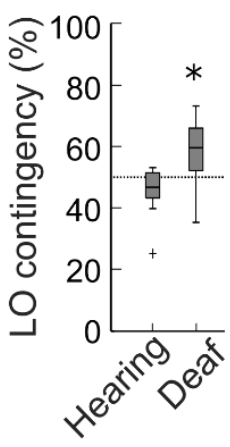

Fig. 2: In hearing birds, the valence of light-off reinforcers is negative. $a, b$, Hearing birds change pitch in the direction of decreasing LO rate, here shown for a low-pitch light-off (LO low) bird (bird b2y2, a) and a LO high bird (bird p6s6, b). Legend as in Fig. 1d, f. c, Histograms of average daily pitch changes in LO high (blue, n=6), LO low (green, $n=6)$, and in no LO birds (gray, $n=12) . d$, The three mixed linear fixed effect terms and their standard errors. The bars indicate the daily change in pitch (d'/day) during baseline (left, $p=0.28$ ), during LO exposure in the direction of increasing LO rate (middle, $p=1.6^{*} 10^{-4}$ ), and

114 in control birds (no LO, right, $p=0.96$ ). e, Average directed pitch changes in LO (hearing) vs subs (deaf) birds. Hearing birds changed their pitch away from the LO zone (decreasing the number of renditions with LO) and deaf birds towards the LO zone (increasing the number of renditions with LO). The error 


\section{The same stimulus aversively reinforces vocal pitch in hearing birds}

We also evaluated adaptive pitch responses in hearing birds. A small number of hearing birds responded aversive in hearing birds. In combination, our findings demonstrate that deafening causes an inversion of affective valence of LO reinforcers.

134 Valence inversion was also signaled by the contrasting effects of light-off on singing rates. Subs birds produced $280 \%$ or 1164 more song motifs on the last three days of substitution than their deaf controls

136 (non-zero fixed effect of light-off, $p=5 * 10^{-4}, D F=58, S E=318$, tstat=3.66, N=20 syllables including 10

137 syllables from controls), suggesting that substitution increases the motivation of deaf birds to sing. 
hearing controls (fixed effect of light-off, $p=0.72, D F=70, S E=564$, tstat $=-0.36, N=24$ birds including 12 control birds).

Deaf and hearing birds exhibited different LO contingencies. While in hearing birds on average $46 \%$ of syllable renditions triggered light-off $(p=0.08$, tstat $=-1.91, \mathrm{df}=11$, two-tailed t-test of hypothesis that LO rate is $50 \%)$, in deaf birds the average LO rate was $58 \%(p=0.04$, tstat $=2.45, d f=9$, two-tailed t-test of

144 hypothesis that LO rate is $50 \%$, Fig. $2 \mathrm{~g}$ ). Thus, only deaf birds diverged from the $50 \%$ expectation set by 145 the previous day.

146 To analyze the sensitivity of birds' vocal response irrespective of whether they were attracted or

147 repelled by light-off, we quantified their magnitude pitch responses as the normalized pitch change per day ( $d^{\prime}$ value) aligned in the direction of global pitch change, implying that the average magnitude

149 change was always a positive number. The daily magnitude pitch change was larger by $135 \%$ in deaf 150 birds than in hearing birds (difference $0.16 d^{\prime} /$ day, $p=0.01$, tstat $=2.73, d f=20, n=12$ hearing and $n=10$ 151 deaf birds, two-tailed t-test), Fig. 2f. Thus, visual feedback is much more salient when it substitutes 152 auditory feedback in deaf animals than as a supplemental feedback in hearing birds.

153 Nevertheless, deaf and hearing birds similarly changed their songs in response to substitution, their 154 pitch changes were confined to a temporal window within roughly $10 \mathrm{~ms}$ of the targeted time window 155 for LO delivery, Fig. S2 and S3.

The inversion in valence does not reflect a preference for darkness in deaf birds

157 A simple explanation of our findings could be that deafness induces an attraction to darkness for

158 whatever reason. This explanation was ruled out after we replaced light-off by light-on stimuli and found 159 strongly appetitive responses to such stimuli in deaf birds $\left(0.71 \pm 0.07 \mathrm{~d}^{\prime} /\right.$ day in the direction of 160 increasing light-on rate (0.61 and $0.77 \mathrm{~d}^{\prime} /$ day), $2 / 2$ birds significantly exceeded spontaneous pitch drift 
161 in control (unsubs) birds, $p<0.05$, two-tailed t-test on pitch changes per day, light-on contingency

$162 \quad 77 \% \pm 8 \%$ (75\% and 79\%), see Methods).

163 A manipulation bonus seems to be required to explain valence reversal

164 Our vocal-light substitution paradigm forms a simple but powerful touchstone for theories of intrinsic

165 motivation because 1) the vocal space of deaf birds is essentially binary (light on vs off), 2) the

166 environment has no intrinsic dynamics (light only depends on pitch), 3) there is no evolutionary

167 adaptation to LO stimuli, and 4) birds have no physiological need to sing a particular pitch (unlike their

168 need of food intake). Despite this simple framework, most models of behavioral learning cannot

169 accommodate valence inversion. In reinforcement learning $(R L)^{21}$, stimuli have either appetitive or

170 aversive effects and standard RL models cannot accommodate valence inversion for example via

171 changes in baseline reward due to deafening ${ }^{22,23}$.

172 Our findings are also incongruent with computational models of directed exploration that involve an

173 exploration bonus for action policies that are either informative ${ }^{12-14}$, diverse ${ }^{8,9}$, or simple ${ }^{24}$, Table 1.

174 These theories have been designed to either improve the efficiency of reinforcement learning models or

175 to model human behavior within a restricted class of multi-armed bandit problems ${ }^{10,12,13}$. In these

176 models, agents choose actions that maximize the information gained about the environment, which is

177 often modeled as an exploration bonus in proportion to the uncertainty of an action's value ${ }^{9,10}$. Yet, in

178 binary (and pitch-symmetric) worlds, knowledge gain is maximal when agents uniformly sample from

179 their action repertoire, implying that such theories predict convergence of LO contingency to $50 \%{ }^{12,13,25}$,

180 which contradicts the divergence we found in subs birds.

\begin{tabular}{|l|l|l|}
\hline $\begin{array}{l}\text { Reinforcement learning } \\
\text { model characteristic }\end{array}$ & Additional info & $\begin{array}{l}\text { Can it explain } \\
\text { valence inversion? }\end{array}$ \\
\hline Changes in baseline reward & Caused by deafening & No \\
\hline Exploration bonus & For informative actions & No \\
\hline
\end{tabular}




\begin{tabular}{|l|l|l|}
\hline & For diverse actions & No \\
\hline & For simple actions & No \\
\hline Manipulation bonus & For impact & Yes \\
\hline
\end{tabular}
impact the environment (such as light off). We introduced such a bias by defining a manipulation bonus $M_{j}$ associated with action $j$. This bonus $M_{j}=D_{K L}\left(\hat{\vartheta}_{0} \| \hat{\vartheta}_{j}\right)$ models the impact of action $j$ in terms of the

Kullback-Leibler divergence between the estimated sensory probability density $\hat{\vartheta}_{j}$ following action $j$ and the same density $\hat{\vartheta}_{0}$ without any preceding action. Let us denote the LO probability following action $j$ by $\hat{\vartheta}_{j}(o f f)$ and the LO probability without acting by $\hat{\vartheta}_{0}(o f f)$. Because $\hat{\vartheta}_{0}(o f f)=0$, it follows that in deaf birds, the impact of action $j$ is given by the Shannon surprise of light on: $M_{j}=-\log \hat{\vartheta}_{j}$ (on). The impact is the larger the more likely the action triggers LO (the smaller $\hat{\vartheta}_{j}(o n)$ ). By experimental design, the impact is nonzero only for a small set of LO actions. An agent that maximizes impact will therefore exhibit a (manipulation) bias towards LO. In hearing birds, by contrast, the sensory environment includes vision and audition. Thanks to auditory feedback, all vocalizations in hearing birds elicit a nonzero impact. Thus, when hearing birds maximize impact, no particular action is singled out, which leads to absence of a manipulation bias towards light off. of an exploration bonus $E_{j}$ (given by information gain), a manipulation bonus $M_{j}$, and an extrinsic

197 punishment $r_{j} \leq 0$ associated with light-off $\left(r_{j}<0\right.$ only in case of light off), Fig. 3a, b. We simulated a 198 simple agent that maximizes $R_{j}$ using $\mathrm{SARSA}^{26,27}$, a standard RL framework. In simulations, we found that when the punishment term $r_{j}$ per LO was such that deaf birds' LO contingency converged to values above and hearing birds' to values below $50 \%$, Fig. $3 d$, the singing preference increased in deaf birds and 201 it decreased in hearing birds, compared to their simulated controls, Fig. 3e, in excellent agreement with 
a

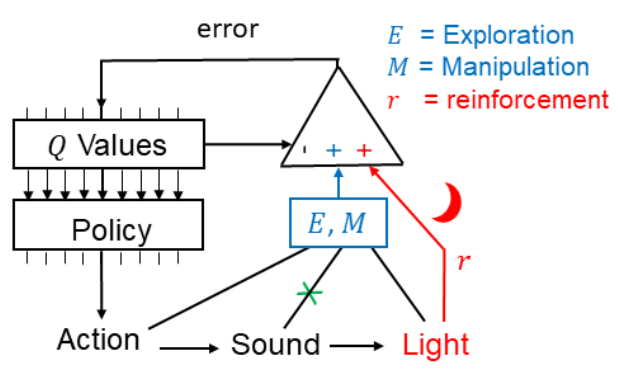

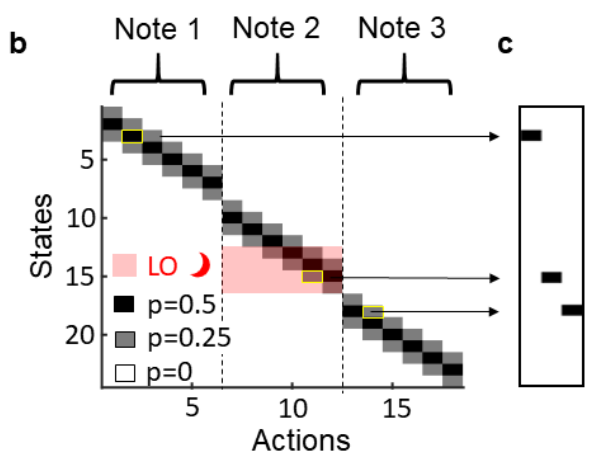

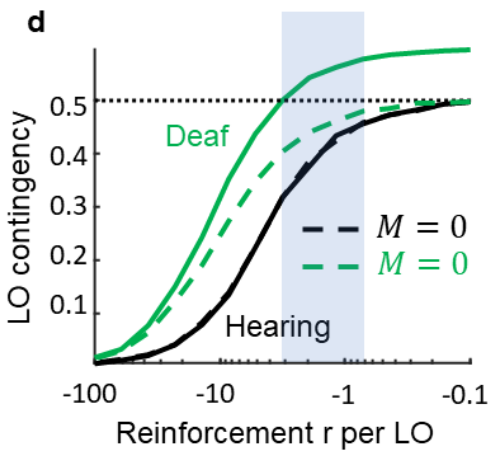

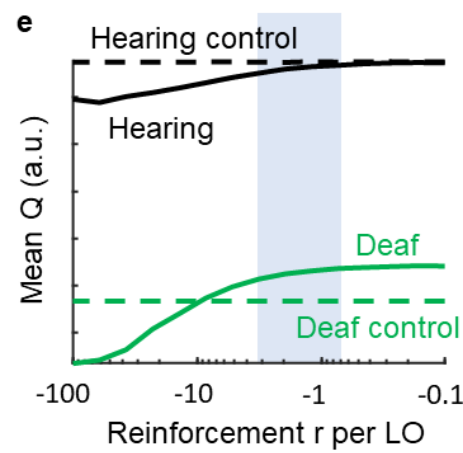

f

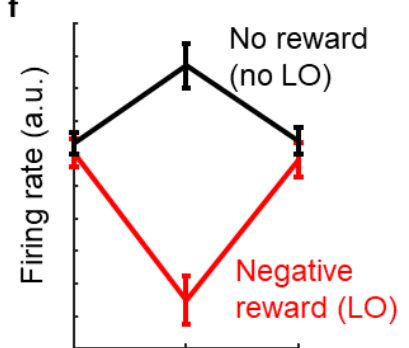

Note 1 Note 2 Note 3

Fig. 3: A manipulation bonus is compatible with valence inversion. $\boldsymbol{a}$, We modeled a simple agent that 
underlying action-state pairs are delimited in yellow in $\boldsymbol{b}$ ). $\boldsymbol{d}$, Hearing birds trigger light-off on less than

$21750 \%$ of syllables for all choices of negative reinforcement $r$ per LO. Deaf birds go above the 50\% LO

218 contingency (green). Without manipulation bonus ( $M=0$, dashed line) this is not the case $(\beta=0)$. ,

Simulated subs birds are more motivated to sing than their controls (unsubs), the mean $Q$ value (green, arbitrary units) for subs is above that of unsubs (dashed green). In hearing birds, the situation is reversed, they are less motivated than their controls. The blue dashed area indicates the plausible reinforcement-per-LO region that qualitatively matches our results. $f$, simulated firing rate of Area-X projecting dopaminergic neurons in hearing birds, modeled as reward prediction errors of a simple $Q$ learning rule ${ }^{26}$. On aversively reinforced trials during Note 2 (modeling a brief LO or an acoustic white noise stimulus), the firing rate decreases (red), whereas on escape trials (no reinforcer, no LO), the firing rate increases (black). Error bars depict standard deviations (across simulated model birds).

\section{Basal ganglia lesions prevent visual reinforcement of pitch}

manipulation bias. Dopaminergic neurons can drive selective pitch changes via their action in Area $\mathrm{X}^{30-32}$,

231 a region homologous to the mammalian basal ganglia ${ }^{18,33}$. To probe for a manipulation bias in Area $\mathrm{X}$, we

232 made irreversible bilateral lesions in Area $\mathrm{X}$ of deaf birds. When these birds were subjected to

233 substituted LO feedback, none of them $(N=5)$ changed pitch in excess to deaf controls ( $p>0.05$ for all

234 birds, two-sampled t-test), Fig. 4a-c. One bird in which the lesion did not overlap with either Area X or

235 LMAN in both hemispheres changed pitch significantly compared to deaf controls $(p=0.01$, two-sampled

236 t-test). In lesioned subs birds, the magnitude of average pitch change per day was smaller than in

237 unlesioned subs birds (difference $-0.22 \mathrm{~d}^{\prime} / \mathrm{day}, \mathrm{p}=0.003$, tstat=-3.62, $\mathrm{df}=12$, two-tailed two-sample t-

238 test), and the daily pitch change in lesioned subs birds was not significantly different from zero (-0.02 
a

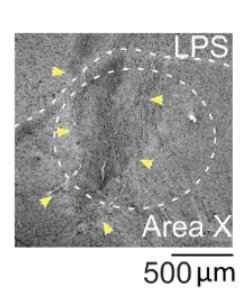

c

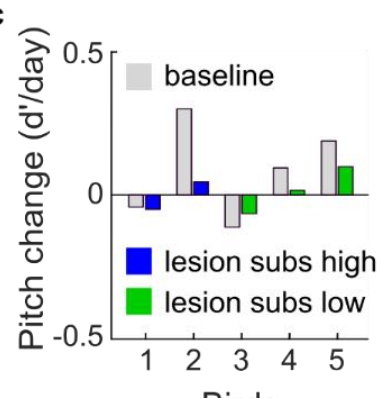

baseline

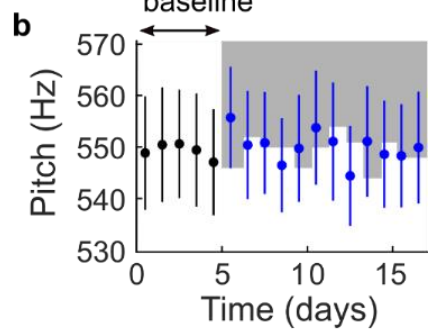

d

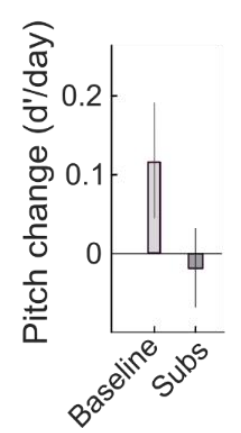

Fig. 4: A basal ganglia pathway is necessary for adaptive responses to substituted feedback. $a$,

\section{Discussion}


253 We found that elimination of auditory feedback induces appetitiveness of an otherwise aversive visual

254 reinforcer of song. This finding helps to refine our understanding of the neural basis of vocal learning.

255 Namely, because targeted changes of vocal skills can occur without hearing, it follows that evaluation of

256 auditory performance is not a prerequisite for vocal plasticity in adulthood, unlike commonly

257 assumed ${ }^{29,33}$. Our findings do not rule out the use of vocal performance for template-based song

258 learning ${ }^{34}$, but they showcase that some forms of vocal learning do not rely on auditory representations

259 of song, but rather on motor representations.

260 This means that the song system is able to assign a cross-modal signal such as light the role of an

261 instructive signal that can selectively bias motor variability. To enable flexible assignment of visual

262 signals (light brightness) to specific motor features (pitch), the visual system must feed into the song

263 system in a computationally powerful way. We find that this cross-modal circuit involves the basal

264 ganglia, which provides some clues as to the underlying neural mechanisms. For one, given that cerebral

265 neurons efferent to the basal ganglia do not even respond to auditory feedback during singing ${ }^{35-37}$, it is

266 unlikely that multimodal visual-vocal neurons are involved in this cross-modal learning flexibility. Rather,

267 a large body of work on the basal ganglia suggests existence of an error-like signal that reinforces time-

268 resolved motor representations of song ${ }^{29,31,38}$. Our work therefore suggests that the avian basal-ganglia

269 part of the song system has evolved to support multimodal learning independent of the sensory

270 modality of reinforcement.

271 We found that substitution uncovers a strong drive to manipulate. This drive better explains our

272 observations than intrinsic motivations such as activity and exploration ${ }^{15}$. Normally, in healthy

273 vocalizers, the need to manipulate is satisfied and does not constrain the brain's valence system.

274 However, when sensory feedback is lacking, this need becomes overwhelming to the point that it can

275 override the valence even of aversive stimuli. This remarkable dictatorship of the manipulation drive 
emphasizes one of the most basic needs associated with motor actions, which is to perceive sensory

277 feedback.

278 By design, the slightly aversive LO events are the only feedback that deaf birds experience, which is why

279 to satisfy their manipulation drive, they prefer it over no response at all ('something' is preferable for a

280 curious agent over 'nothing'). One function of song in birds may thus be to exert an influence on the

281 environment to signal the singer's presence, even if confirmed only by visual feedback as in our

282 experiment. Birds' tendency to avoid overlap in their vocalizations ${ }^{40}$ is evidence for such a need of

283 undisturbed signaling.

284 In humans, there exists a compelling analogy of the remarkable transformation of affective stimulus

valence we observed. Namely, a manipulation drive shows up during boredom, which can prompt subjects to display behaviors that evidence paradoxical preference of otherwise aversive stimuli ${ }^{41,42}$.

287 Lacking an alternative, subjects prefer an unpleasant experience rather than none at all.

Regarding substitution as a therapy, perhaps the frustration experienced by users of substitution devices

289 is linked with the level of uncontrollability of the substituting input. Our findings suggest that users of

290 substitution devices might avoid motor actions unless these elicit some form of substituting sensory

291 feedback. Although the intention behind a substitution system usually is to maximize the conveyed

292 information, to merely maximize information can conflict with a manipulation bias that may draw the

293 use of a device away from its intended purpose.

294 We suspect that substitution devices are preferred when they provide feedback about motor actions,

295 making subjects feel empowered through the new sense. By designing substitution systems as part of

296 closed sensorimotor loops ${ }^{4}$, the systems may stimulate motor learning, which can be fun as in tennis

297 practice or piano playing rather than strenuous as in learning a foreign language (analogies between

298 learning to make use of substituted input and reading have been drawn ${ }^{1}$ ). Perhaps, acceptance of 
substitution devices would also increase when training setups are designed to let subjects predictably manipulate the substituting sensory input, in line with insights from interviews conducted with users of substitution devices ${ }^{43}$. desirable actions. Binary feedback signals are known to promote robust motor learning ${ }^{44}$. For example, the blind might benefit from a signal that reports the inverse distance of a hand to an object of choice.

feedback when their variable speech agrees with signals of high comprehensibility; such feedback could be provided as visual signal (e.g. displayed in augmented reality devices) or as vibro-tactile signal ${ }^{45}$.

311 electrical stimulation of downstream neurons. While neuroprosthetic closed-loop systems have only

312 recently started to be explored in the sensory domain ${ }^{46}$, closed-loop systems are very common in the 313 motor domain ${ }^{47}$, where animal models have played a crucial role in the development of a wide range of 314 those systems ${ }^{47,48}$. Closed-loop motor systems achieve better performance than open-loop systems ${ }^{47,48}$ 315 and there is a distinct performance benefit of high feedback rates ${ }^{49}$. These facts lend support to the idea 316 that sensory neuroprosthetic systems will also benefit from closed-loop design. The zebra finch as an

317 animal model may lend itself to exploring the closed-loop benefits of sensory neuroprosthetics.

318 Key to our findings is that songbirds have a strong manipulation drive. Abstracted as a principle of 319 software agents, such a drive can serve some computational functions. Conceptually, manipulation 320 seeking can be preferable to knowledge seeking because the latter is uninformative about relevance. 321 For example, a manipulation drive can prevent an agent from getting stuck in front of a computer screen 
322 displaying random stimuli, which would otherwise be the most absorbing stimulus for a purely

323 knowledge-driven agent that does not distinguish between self-generated and external stimuli. It is

324 therefore not surprising that concepts such as manipulation and impact are gaining in importance in

325 machine learning. In a recent curiosity-driven RL approach, it was found that a focus of actions on self-

326 generated sensory feedback can dramatically expedite learning progress ${ }^{50}$.

327 Further impulses for understanding the motivational drives of spontaneous behaviors are highly needed.

328 We propose that sensory substitution is a promising paradigm not just to experimentally characterize

329 the motivation to manipulate, but also to dissect the neural representations of affective valence ${ }^{51}$ and to

330 probe how substituting input is integrated into an existing circuit on the level of single cells, which so far

331 is only understood on the level of brain areas ${ }^{1,39}$. Because the manipulation drive seems to have access

332 to cross-modal learning mechanisms that are as fast and efficient as those of normal motor learning,

333 sensory substitution and the manipulation drive it reveals may provide a glimpse on some of the

334 enabling factors of evolutionary adaptations.

\section{Acknowledgments}

337 We thank Florian Engert, Maneesh Sahani, and Georg Martius for helpful discussions and Benjamin

338 Grewe, Thomas Tomka, and Catherine del Negro for helpful comments on the manuscript.

339 Funding support was provided by the Swiss National Science Foundation (Project 31003A_182638).

340 The authors declare no competing financial interests.

341 Data and scripts can be accessed at the ETH Research Collection.

\section{$342 \quad$ Author Contributions}




\section{Declaration of Interests}

The authors declare no competing interests.

\section{Materials and Methods}

Subjects and song recordings: We used 50 adult male zebra finches (Taeniopygia guttata) raised in our breeding facilities in Zürich (Switzerland) and Orsay (France). At the beginning of the experiment, birds were between 90 and 200 days old. During the experiment, birds were housed individually in soundattenuating recording chambers on a $14 / 10 \mathrm{~h}$ day/night cycle. Access to food and water was provided ad libitum. After 2-5 days of familiarization in the experimental environment, birds resumed singing at a normal rate. Songs were recorded with a wall-attached microphone, band-pass filtered, and digitized at a sampling rate of $32 \mathrm{kHz}$. All experimental procedures were approved by the Veterinary Office of the

357 Canton of Zurich and by the French Ministry of Research and the ethical committee Paris-Sud and Centre 358 (CEEA N59, project 2017-12).

359 Visual substitution of pitch: To provide pitch substitution, we ran a custom LabVIEW (National

360 Instruments, Inc.) program. We targeted a harmonic syllable using a two-layer neural network trained 361 on a subset of manually clustered vocalizations. We evaluated pitch (fundamental frequency) in a 16-ms 362 window at a fixed delay after the syllable detection point (which occurred at a roughly constant time lag 363 after syllable onset). We estimated pitch using the Harmonic Product Spectrum algorithm ${ }^{52}$, our code is 364 published at the ETH Research Collection, also in the Matlab (Mathworks Inc.) language. 
We provided pitch substitution by switching off the light (using a relay) in the sound recording chamber after a delay of $12 \mathrm{~ms}$ and for a duration in the range of 100 to $500 \mathrm{~ms}$. Two birds were put in dim light and substitution was provided turning on an additional light instead of switching off the light. We substituted either high or low pitch, depending on a manually set threshold.

To cumulatively drive pitch of the targeted syllable away from baseline, every morning, we adjusted the pitch threshold to the median pitch value from the previous day based on all noncurated neural network detections (in 24/328 days from 15/29 birds, we did not set the threshold to the median value because of a software crash on the previous day). In 15 birds ( 6 hearing and 9 deaf, among which2 were Area X

373 lesioned birds and 1 missed lesion) we delivered substitution on high-pitched syllable renditions, and in

37415 birds (6 hearing and 9 deaf, among which 3 were Area-X lesioned birds) on low-pitched syllable

375 renditions. Subs birds were deaf birds with LO substitution, unsubs birds were deaf and unsubstituted

376 birds; LO birds were hearing and subjected to LO, and no LO birds were hearing and not subjected to

377 LO.

Surgeries: Before the onset of surgery, we provided analgesia with the nonsteroidal anti-inflammatory drug carprofen (2-4 mg/kg, Norocarp, ufamed AG, Sursee, Switzerland) given intra muscularly (IM). Birds were deeply anesthetized using isoflurane (1.5-3 \%) and placed in a stereotaxic apparatus. We applied the antiseptic povidone-iodine (Betadine, Mundipharma Medical Company, Basel, Switzerland) to the skin at the incision site, followed by the local anaesthetic lidocaine in gel form ( $5 \%$, EMLA, AstraZeneca AG, Zug, Switzerland).

Deafening procedure: In the stereotax, the head angle formed by the flat part of the skull above the beak and the table was set at $90^{\circ}$. The skin was opened above the hyoid bone and the neck muscles were gently pushed back to expose the semi-circular canals. A hole was made in the skull to access the inner ear below the semi-circular canals. The cochlea was visually identified based on the surrounding 
bone structure and a small hole was made with forceps into the cochlear base. We removed the cochlea the lagenas to document the success of the procedure, Fig. $1 \mathrm{~b}$.

Area X lesions: We set the head angle formed by the flat part of the skull above the beak and the table to $35^{\circ}$ and drilled a window into the skull above Area X. Area X was localized based on stereotaxic coordinates and identified through presence of tonically firing neurons, recorded with a 0.6-1.7 $\mathrm{M} \Omega$ tungsten electrode attached to a vertical manipulator. We injected in each hemisphere $1 \mu \mathrm{l}$ of ibotenic acid (Tocris) near the center of Area X. Injection sites were located on average 1.5-1.9 mm medial-lateral (ML), 5.5-6.0 mm anterior-posterior (AP), and 2.8-3.5 $\mathrm{mm}$ dorsal-ventral (DV) from the bifurcation of the midsagittal sinus (lambda). Injections were performed using a borosilicate glass pipette (BF-120-69-10, Sutter instrument) pulled with a Picospritzer (Parker Inc.) and broken with forceps to a tip diameter of about $10 \mu \mathrm{m}$.

Histology: At the end of the experiment, birds were euthanized with an overdose of intraperitoneal

401 injection of sodium pentobarbital ( $200 \mathrm{mg} / \mathrm{kg}$, Esconarkon, Streuli Pharma AG, Uznach, Switzerland) and 402 intracardially perfused with $4 \%$ paraformaldehyde (PFA) before brains were removed for histological

403 examination. Brains were rinsed in a $0.01 \mathrm{M}$ phosphate buffer solution. The hemispheres were 404 separated from each other, glued on a metal plate, and embedded in 3\% agar. Sagittal slices of $80 \mu \mathrm{m}$ 405 thickness were cut with a Thermo Microm HM650V microtome and mounted on slides for Nissl staining.

406 Statistical pitch analysis: We curated the neural network detections manually by visually removing 407 misdetections (detection of noises or timepoints in the song that did not correspond to the selected target syllable). We quantified the effects of light-off (LO) on pitch using d-prime values $d_{i, j}^{\prime}$ :

$$
d_{i, j}^{\prime}=\frac{\bar{p}_{j}-\bar{p}_{i}}{\sqrt{\frac{1}{2}\left(\sigma_{i}^{2}+\sigma_{j}^{2}\right)}}
$$


where $\bar{p}_{i}$ and $\bar{p}_{j}$ are the mean pitches on days $i$ and $j$, and $\sigma_{i}^{2}$ and $\sigma_{j}^{2}$ are the variances, respectively.

411 LO start criterion: LO started after at least 5 days of stable singing $\left(\left|d_{i-4, i}^{\prime}\right|<0.5\right.$ with $i$ being the last

412 day of baseline); in two birds, LO started earlier and in one bird, LO started later because of technical

413 issues and unforeseen scheduling constraints. In deaf (subs) birds, LO started on average 15 days after

414 deafening (range 7 to 33 days) and in hearing (LO) birds it started after at least 7 days in isolation.

415 LO end criterion: We ended the LO paradigm when the absolute mean pitch change (relative to

416 baseline) either exceeded at least $2.5 d^{\prime}$ or when it stabilized near zero, which was defined as $\left|d_{i-4, i}^{\prime}\right|<$

$417 \quad 0.5$ with the index $i$ referring to the last day of light off (in one bird, we ended substitution before this

418 criterion was reached because the song degraded too much for reliable syllable detection). The duration

419 of the substitution paradigm did not differ significantly between birds in the hearing and deaf groups ( $p$

$420=0.39$, two-tailed two-sample t-test, mean hearing $=13$ days, mean deaf $=11$ days). Thus, the observed

421 differences between hearing and deaf birds in Fig. 2e, $\mathrm{f}$ were not due to differences in time spent in the

422 experimental chamber.

423 The average daily pitch change $\overline{d_{L O}^{\prime}}$ during substitution in each animal (Figs. $1 \mathrm{~g}$ and $2 \mathrm{c}$ ) we quantified

424 as $\overline{d^{\prime}{ }_{L O}}=\left\langle d_{i-1, i}^{\prime}\right\rangle_{i}$, where the angle brackets denote averaging across all days $i$ with LO (starting from

425 the second day).

426 Similarly, the average daily pitch change $\overline{d^{\prime}}$ during the baseline period in each animal (light grey bars

427 in Figs. $1 \mathrm{~g}$ and $2 \mathrm{c}$ ) we quantified as $\overline{d^{\prime}{ }_{B}}=\left\langle d_{i-1, i}^{\prime}\right\rangle_{i}$, where the average runs across the last 4 days $i$

428 before LO.

429 Magnitude pitch change: We assessed the magnitude pitch change in each bird irrespective of its

430 preference (attraction or repulsion by LO). To discount for preference, we first defined the global

431 direction $\delta$ of pitch change during substitution as $\delta=\operatorname{sign}\left(d_{b, l}^{\prime}\right)$, where $b$ is the last day of baseline and 
$432 l$ is the last day of LO exposure ( $\delta$ corresponds to the direction of the colored bars in Figs. $1 \mathrm{~g}$ and $2 \mathrm{c}$ ).

433 Thus, if birds shifted pitch upward towards higher values, $\delta=1$, and if birds shifted pitch down, $\delta=$

$434-1$. In each animal, we computed the mean aligned pitch change $\overline{a^{\prime}}$ during substitution as the average

435 daily change $d_{i-1, i}^{\prime}$ multiplied with $\delta: \overline{a^{\prime}}=\left\langle\delta \cdot d_{i-1, i}^{\prime}\right\rangle_{i},\left(i=6, \ldots\right.$, end). Fig. $2 \mathrm{f}$ shows $\overline{a^{\prime}}$ averaged over all

436 birds. For control birds (unsubs, no LO), the direction of change $\delta$ was calculated analogously.

437 Sound features other than pitch: To test whether substitution-induced sound feature changes of the

438 target syllable were specific to pitch, we inspected sound features including syllable duration, amplitude

439 modulation (AM), frequency modulation (FM), and entropy. Syllable duration was defined by the

440 interval between consecutive threshold crossings of RMS sound waveform, the threshold for each

441 animal was kept constant for all days analyzed. AM, FM, and entropy were computed as means over the

442 entire syllable. For the analysis, we combined low-pitch and high-pitch substituted birds, all feature

443 values in low-pitch birds were first multiplied by -1 to account for the anti-symmetry between their

444 treatments. As a group, we compared the feature $d^{\prime}$ values between the last LO day and the last day of

445 baseline (paired two-tailed t test), Supplementary Fig. S1a.

Control birds: To evaluate whether an individual bird responded to substitution, we compared the daily

447 pitch changes $\left\{d_{i-1, i}^{\prime}\right\}_{i \in L O}$ of its targeted syllable to daily pitch changes of harmonic syllables (containing

448 a harmonic part longer than $100 \mathrm{~ms}$ ) in many control birds (not exposed to LO). In subs birds, the control

449 group was formed by 12 harmonic syllables in 9 deaf animals, and in LO birds, the control group was

450 formed by 12 harmonic syllables in 12 hearing birds. To account for possible pitch drifts caused by

451 deafening or by time spent in the experimental chamber, the time window for pitch analysis in unsubs

452 birds was matched to the substitution period in the subs bird, i.e., the first day analyzed in control birds

453 occurred at the same time lag after deafening as the first LO day. Also, the number of days analyzed was

454 identical in the subs bird and in control birds (same for LO and no-LO birds). We paired a subs bird only 
to controls that produced more than 100 song motifs on each day during the matched time periods. Two

456 unsubs birds had to be excluded because they produced fewer than 100 renditions on days 11 and 12

457 after deafening (these birds could not be time-matched to any subs bird, resulting in 10 harmonic

458 syllables from 7 control birds).

Pitch response testing: To test whether an individual bird significantly changed its pitch in response to

LO, we compared all its daily pitch changes during LO to all daily pitch changes in control birds in

For the population analysis, we compared daily pitch changes in all subs/LO birds against all unsubs/no-

LO controls. We randomly paired 10 syllables in control birds (dark grey bars in Fig. $1 \mathrm{~g}$ ) with the 10

syllables in subs birds (under the constraint that analysis days could be temporally matched). The pairing is depicted in Fig. 1g such that target syllable 11 was paired with subs bird 1, target syllable 12 with subs bird 2, etc. We did the same for the 12 syllables in LO birds in Fig. 2c, i.e., target syllable 13 was paired with LO bird 1, target syllable 14 was paired with LO bird 2, etc. All pairings were time-matched, i.e., the early (baseline, light gray bars in Fig. 1g) and late time periods in controls were defined according to the baseline and LO periods in the treated bird.

471

472 Linear mixed effect model: To test whether subs/LO birds exhibited a common direction of pitch change

473 (either towards LO or away from it), we modeled daily pitch changes $d_{i-1, i}^{\prime j}$ in bird $j$ in response to LO 474 with a linear mixed effect model:

$$
d_{i-1, i}^{\prime j}=b \vartheta_{i}+a \theta_{i}+d \varphi_{i}+r_{j}
$$

476 where the three fixed effect terms $b, a$, and $d$ common to all birds were: the daily pitch change $b$ during 477 baseline $\left(\vartheta_{i}=1\right.$ if day $i$ is during baseline and $\vartheta_{i}=0$ otherwise), the pitch drift $a$ without LO $\left(\theta_{i}=1\right.$ in 
478

479

480

481

482

483

484

485

control birds if days $i$ and $i-1$ occurred after baseline and $\theta_{i}=0$ otherwise), and the daily pitch change $d$ caused by LO ( $\varphi_{i}=1$ for LO high and $\varphi_{i}=-1$ for LO low birds, provided both days $i-1$ and $i$ were LO days). The $r_{j}$ are zero-mean Gaussian noise terms that account for variability among birds. We separately fitted linear mixed effect models to deaf and to hearing birds.

When we reduced the model to two fixed effects (combining the terms $b$ and $a$ into a single term describing spontaneous pitch drift during both baseline in LO-treated birds and all days in control animals) we found the results displayed in Fig. $1 \mathrm{~h}$ and Fig. $2 \mathrm{~d}$ to be qualitatively unchanged.

Song degradation: To assess song degradation caused by deafening (Supplementary figure S1b-e), we inspected non-targeted syllables, comparing renditions at the beginning and the end of the experiment. Tschida and Mooney showed that both entropy and entropy variance significantly change after deafening ${ }^{20}$. Mean entropy is a measure of syllable noisiness and variance entropy of syllable complexity. To follow suit and inspect mean and variance entropy, we first semi-automatically clustered all (non-targeted) syllables using a nearest neighbor approach in the spectrogram domain. We only considered syllables that were sung more than 100 times on each day ( 21 syllables in hearing birds and 18 syllables in deaf birds). We calculated for each syllable type the magnitude mean-entropy change as the absolute difference in mean entropy between the last day before deafening and the first day after LO ended. For hearing birds, we chose the first day analyzed such that the duration of the analysis window matched the window in deaf birds. As a result, the intervals between the first and last day of the experiment did not significantly differ between birds in the hearing and deaf groups $(p=0.86$, twotailed two-sample t-test, mean hearing $=32$ days, mean deaf $=31$ days). Thus, differences between hearing and deaf birds in Supplementary Fig. S1b-e were not due to differences in time spent in the recording chamber. 
501 In agreement with Tschida and Mooney, we found that deaf birds have a larger magnitude variance-

502 entropy change than hearing birds (difference $0.32, p=9.3 * 10^{-4}$, tstat $=3.60, \mathrm{df}=37$, two-tailed two-

503 sample t-test, Suppl Fig. S1c). However, we found no difference in magnitude mean-entropy change

504 ( $p=0.61$, Suppl Fig. S1b). Note that Tschida and Mooney did not perform time-matched comparisons

505 against a group of hearing birds as we did, but they compared entropy to baseline measurements taken

506 before deafening, implying that mean entropy changes in their study could have been caused by birds'

507 gradual adaptation to the recording chamber, irrespective of the deafening procedure.

508

For non-targeted syllables, we calculated the pitch coefficient of variation $C V_{i}$ on day $i$ as $C V_{i}=100 \frac{\sigma_{i}}{\bar{p}_{i}}$.

As we had done for targeted syllables, we calculated the pitch within a fixed $16 \mathrm{~ms}$ window during a harmonic part of the syllable (provided the latter existed, i.e., a harmonic part was found in 9/18 syllables in deaf animals and in 9/21 syllables in hearing animals). The difference between the coefficients of variation on the last day of deafening and on the first day after LO is shown in Suppl Fig. S1d. In deaf birds, this difference was larger than in hearing birds.

To compute spectral changes due to deafening we performed a bias-variance decomposition. To calculate spectrograms, we first tapered the sound waveform using a Hamming window of 512 samples. The windowed signal was transformed into a linear-power sound spectrogram using the discrete fast Fourier transform computed over segments of 512 samples and nonoverlaps of 128 samples (corresponding to $4 \mathrm{~ms}$ ). The log-power sound spectrogram was then obtained by taking the natural logarithm of the linear-power sound spectrogram after adding an offset of 0.1 (corresponding roughly to the $75^{\text {th }}$ percentile). We computed the spectrograms of non-targeted syllables within a time window defined by the duration of the shortest syllable rendition. To achieve robustness to low-frequency noise present in the recordings, we ignored the lowest 10 frequency bins corresponding to a frequency cutoff at $625 \mathrm{~Hz}$. The spectrogram bias of a particular syllable was defined as the Euclidean distance between 
524 the average spectrograms on two separate days: on the last day before deafening and the first day after

525 the end of the LO period. The spectrogram variance was defined as the average pixel-wise variance on a 526 given day. There was no significant difference between hearing and deaf birds in terms of either

527 spectrogram bias or variance (bias: $p=0.45$, tstat $=0.77, d f=39$, variance: $p=0.32$, tstat $=-1.01$, $d f=39$, two-

528 tailed two-sample t-test, Suppl Fig. S1e and f). Thus, the substitution period was too short to lead to a

529 major spectral song degradation.

531 Localization of pitch changes: Next we assessed the temporal dynamics of pitch changes in response to

532 light off. We computed pitch traces over the entire syllable in a sliding window of $16 \mathrm{~ms}$ and plotted

533 their temporal statistics at a time resolution of $1 \mathrm{~ms}$, supplementary Fig. S2. In each bird, to compare

534 pitch traces from the last day of light off with traces from the last day before light off, we computed d'

535 values between the two distributions at 1-ms time scale relative to the window of LO delivery,

536 Supplementary Fig. S3. For bird 1, the syllable detection point was not stable and over the experiment

537 the pitch window slightly shifted relative to syllable onset (see Supplementary Fig S2a, bird 1). To avoid

538 the visualization of spurious pitch changes due to syllable detection jitter, we corrected for this temporal

539 jitter before plotting pitch difference traces across the LO period in individual birds and their averages,

540 Supplementary Fig. S3 (we aligned all syllables to their onsets and defined the pitch window at the mean

541 time lag of all windows on the day before light off). 


\section{Bibliography}

1. Deroy, O. \& Auvray, M. Reading the World through the Skin and Ears: A New Perspective on Sensory Substitution. Front. Psychol. 3, 457 (2012).

2. Bach-y-Rita, P., Collins, C. C., Saunders, F. A., White, B. \& Scadden, L. Vision substitution by tactile image projection. Nature 221, 963-964 (1969).

3. von Melchner, L., Pallas, S. L. \& Sur, M. Visual behaviour mediated by retinal projections directed to the auditory pathway. Nature 404, 871-876 (2000).

4. Maidenbaum, S., Abboud, S. \& Amedi, A. Sensory substitution: closing the gap between basic research and widespread practical visual rehabilitation. Neurosci. Biobehav. Rev. 41, 3-15 (2014).

5. Striem-Amit, E., Cohen, L., Dehaene, S. \& Amedi, A. Reading with sounds: sensory substitution selectively activates the visual word form area in the blind. Neuron 76, 640-652 (2012).

6. Galea, J. M., Mallia, E., Rothwell, J. \& Diedrichsen, J. The dissociable effects of punishment and reward on motor learning. Nat. Neurosci. 18, 597-602 (2015).

7. Tye, K. M. Neural circuit motifs in valence processing. Neuron 100, 436-452 (2018).

8. Daw, N. D., O'Doherty, J. P., Dayan, P., Seymour, B. \& Dolan, R. J. Cortical substrates for exploratory decisions in humans. Nature 441, 876-879 (2006).

9. Sutton, R. S. in Machine learning proceedings 1990 216-224 (Elsevier, 1990). doi:10.1016/B978-155860-141-3.50030-4

10. Schulz, E. \& Gershman, S. J. The algorithmic architecture of exploration in the human brain. Curr. Opin. Neurobiol. 55, 7-14 (2019).

11. Daw, N. D., Niv, Y. \& Dayan, P. Uncertainty-based competition between prefrontal and dorsolateral striatal systems for behavioral control. Nat. Neurosci. 8, 1704-1711 (2005).

12. Choshen, L., Fox, L. \& Loewenstein, Y. Dora the explorer: Directed outreaching reinforcement action-selection. arXiv preprint arXiv:1804.04012 (2018).

13. Little, D. Y. \& Sommer, F. T. Learning and exploration in action-perception loops. Front. Neural Circuits 7, 37 (2013).

14. Martius, G., Der, R. \& Ay, N. Information driven self-organization of complex robotic behaviors. PLoS One 8, e63400 (2013).

15. White, R. W. Motivation reconsidered: The concept of competence. Psychol. Rev. 66, 297-333 (1959). 
16. Nelson, J. D. Finding useful questions: on Bayesian diagnosticity, probability, impact, and information gain. Psychol. Rev. 112, 979-999 (2005).

17. Tumer, E. C. \& Brainard, M. S. Performance variability enables adaptive plasticity of "crystallized" adult birdsong. Nature 450, 1240-1244 (2007).

18. Ali, F. et al. The basal ganglia is necessary for learning spectral, but not temporal, features of birdsong. Neuron 80, 494-506 (2013).

19. Konishi, M. The Role of Auditory Feedback in the Control of Vocalization in the White-Crowned Sparrow. Ethology (1965).

20. Tschida, K. A. \& Mooney, R. Deafening drives cell-type-specific changes to dendritic spines in a sensorimotor nucleus important to learned vocalizations. Neuron 73, 1028-1039 (2012).

21. Baron, A. \& Galizio, M. Positive and negative reinforcement: Should the distinction be preserved? Behav. Anal. 28, 85-98 (2005).

22. Williams, R. J. Simple statistical gradient-following algorithms for connectionist reinforcement learning. Mach Learn 8, 229-256 (1992).

23. Weaver, L. \& Tao, N. The optimal reward baseline for gradient-based reinforcement learning. Proceedings of the Seventeenth conference on ... (2001).

24. Rubin, J., Shamir, O. \& Tishby, N. in Decision Making with Imperfect Decision Makers (eds. Guy, T. V., Kárný, M. \& Wolpert, D. H.) 28, 57-74 (Springer Berlin Heidelberg, 2012).

25. Klyubin, A. S., Polani, D. \& Nehaniv, C. L. Empowerment: A Universal Agent-Centric Measure of Control. in 2005 IEEE Congress on Evolutionary Computation 128-135 (IEEE, 2005). doi:10.1109/CEC.2005.1554676

26. Watkins, C. J. C. H. \& Dayan, P. Q-learning. Mach Learn 8, 279-292 (1992).

27. Rummery, G. A. \& Niranjan, M. On-line Q-learning using connectionist systems. (mi.eng.cam.ac.uk, 1994).

28. Schultz, W., Dayan, P. \& Montague, P. R. A neural substrate of prediction and reward. Science 275, 1593-1599 (1997).

29. Gadagkar, V. et al. Dopamine neurons encode performance error in singing birds. Science 354, 1278-1282 (2016).

30. Hisey, E., Kearney, M. G. \& Mooney, R. A common neural circuit mechanism for internally guided and externally reinforced forms of motor learning. Nat. Neurosci. 21, 589-597 (2018).

31. Xiao, L. et al. A basal ganglia circuit sufficient to guide birdsong learning. Neuron 98, 208-221.e5 (2018). 
606

607

608

609

610

611

612

613

614

615

616

617

618

619

620

621

622

623

624

625

626

627

628

629

630

631

632

633

634

635

636

637

32. Kearney, M. G., Warren, T. L., Hisey, E., Qi, J. \& Mooney, R. Discrete evaluative and premotor circuits enable vocal learning in songbirds. Neuron (2019). doi:10.1016/j.neuron.2019.07.025

33. Andalman, A. S. \& Fee, M. S. A basal ganglia-forebrain circuit in the songbird biases motor output to avoid vocal errors. Proc. Natl. Acad. Sci. USA 106, 12518-12523 (2009).

34. Konishi, M. The role of auditory feedback in birdsong. Ann. N. Y. Acad. Sci. 1016, 463-475 (2004).

35. Kozhevnikov, A. A. \& Fee, M. S. Singing-related activity of identified HVC neurons in the zebra finch. J. Neurophysiol. 97, 4271-4283 (2007).

36. Leonardo, A. Experimental test of the birdsong error-correction model. Proc. Natl. Acad. Sci. USA 101, 16935-16940 (2004).

37. Hamaguchi, K., Tschida, K. A., Yoon, I., Donald, B. R. \& Mooney, R. Auditory synapses to song premotor neurons are gated off during vocalization in zebra finches. Elife 3, e01833 (2014).

38. Fee, M. S. \& Goldberg, J. H. A hypothesis for basal ganglia-dependent reinforcement learning in the songbird. Neuroscience 198, 152-170 (2011).

39. Dehaene, S., Cohen, L., Morais, J. \& Kolinsky, R. Illiterate to literate: behavioural and cerebral changes induced by reading acquisition. Nat. Rev. Neurosci. 16, 234-244 (2015).

40. Benichov, J. I., Globerson, E. \& Tchernichovski, O. Finding the beat: from socially coordinated vocalizations in songbirds to rhythmic entrainment in humans. Front. Hum. Neurosci. 10, 255 (2016).

41. Nederkoorn, C., Vancleef, L., Wilkenhöner, A., Claes, L. \& Havermans, R. C. Self-inflicted pain out of boredom. Psychiatry Res. 237, 127-132 (2016).

42. Bench, S. W. \& Lench, H. C. Boredom as a seeking state: Boredom prompts the pursuit of novel (even negative) experiences. Emotion 19, 242-254 (2018).

43. Hamilton-Fletcher, G., Obrist, M., Watten, P., Mengucci, M. \& Ward, J. I always wanted to see the night sky" blind user preferences for sensory substitution devices. in Proceedings of the $2016 \mathrm{CHI}$ Conference on Human Factors in Computing Systems - CHI' ' '16 2162-2174 (ACM Press, 2016). doi:10.1145/2858036.2858241

44. Shmuelof, L. et al. Overcoming motor "forgetting" through reinforcement of learned actions. J. Neurosci. 32, 14617-14621 (2012).

45. Novich, S. D. \& Eagleman, D. M. Using space and time to encode vibrotactile information: toward an estimate of the skin's achievable throughput. Exp. Brain Res. 233, 2777-2788 (2015).

46. Prsa, M., Galiñanes, G. L. \& Huber, D. Rapid Integration of Artificial Sensory Feedback during Operant Conditioning of Motor Cortex Neurons. Neuron 93, 929-939.e6 (2017). 
47. Moxon, K. A. \& Foffani, G. Brain-machine interfaces beyond neuroprosthetics. Neuron 86, 55-67 (2015).

48. Wright, J., Macefield, V. G., van Schaik, A. \& Tapson, J. C. A Review of Control Strategies in ClosedLoop Neuroprosthetic Systems. Front. Neurosci. 10, 312 (2016).

642 49. Shanechi, M. M. et al. Rapid control and feedback rates enhance neuroprosthetic control. Nat.

$643 \quad$ Commun. 8, 13825 (2017).

644 50. Pathak, D., Agrawal, P., Efros, A. A. \& Darrell, T. Curiosity-Driven Exploration by Self-Supervised Prediction. in 2017 IEEE Conference on Computer Vision and Pattern Recognition Workshops (CVPRW) 488-489 (IEEE, 2017). doi:10.1109/CVPRW.2017.70

51. Berridge, K. C. Affective valence in the brain: modules or modes? Nat. Rev. Neurosci. 20, 225-234 (2019).

52. Noll, A. M. Pitch Determination of Human Speech by the Harmonic Product Spectrum, the Harmonic Sum Spectrum and a Maximum Likelihood Estimate. in Proceedings of the Symposium on Computer Processing in Communications, Vol. XIX 779-797 (1970). 